\title{
A Novel Method for Petri Net MODELING
}

\author{
Chen-xi SHAO ${ }^{1,3}$, Er-hui ZHOU ${ }^{1,2}$, Zhen-zhong $\mathrm{SHAO}^{4}$, Wei-hua \\ $\mathrm{WANG}^{5}$, Xue-feng WU ${ }^{1}$ \\ ${ }^{1}$ Department of Computer Science and Techmology, University of Science and \\ Technology of China, Hefei, 230026, China \\ cxshao@ustc.edu.cn, xfwu@ustc.edu.cn \\ ${ }^{2}$ Information Center of Hospital 152 of PLA, Pingdingshan 467000, China \\ ehzhou@mail.ustc.edu.cn \\ ${ }^{3}$ Anhui Province Key Laboratory of Software in Computing and Communication, \\ Hefei 230027, China \\ ${ }^{4}$ Jianghuai Automobile Co., Ltd. Hefei, 230022, China \\ v5vv5v@163. com \\ ${ }^{5}$ Liwei Automobile Oil-pump Co., Ltd. Anhui, Hefei 230041, China \\ 2285684217 @qq. com
}

\begin{abstract}
Message Sequence Chart (MSC) is a graphical trace language for describing the communication behaviour of distributed systems. In order to build a Petri Net model of a complex system, this paper introduces a strategy to build a Petri Net model by using MSC. The algorithm of translating the MSC model to Petri Net model is also given in this paper. We can get the Petri Net model and improve it by translating the model of MSC. By analyzing the Petri Net model, it is proved to be safe and reliable.
\end{abstract}

\section{KEYWORDS}

modeling/simulation; Message Sequence Charts(MSC); Petri Net

\section{INTRODUCTION}

Petri nets is a graphic representation of the combination model, has the merits of intuitive, simple and easy, for describing and analyzing of concurrent phenomena have unique advantage. Of the existing modeling methods, the Petri Net modeling has got well developed and widely applied. It not only has the strict mathematical basis, but also has a lot of analytical and simulation tools. However, the traditional method for building a Petri Net model requires highly for the modeling staff: They must be good at mathematics and familiar to the structure and function of the complex system.

In order to simplify the Petri Net modeling of a complex system, this paper present a new modeling method, which uses Message Sequence Charts(MSC) to model the research objects at first, then translate the MSC model to Petri Net model with the corresponding algorithm. This method can take full advantage of MSC which is simple, intuitionistic and easy to modify, and Natarajan Meghanathan, et al. (Eds): ITCS, SIP, JSE-2012, CS \& IT 04, pp. 71-78, 2012. 
can be used to build the Petri Net model for analysis and modeling. By analyzing the Petri Net model, it is proved to be safe and reliable.

\section{Message Sequence CharTS ${ }^{[1,2]}$}

MSC is a good graphical language for describing the communication behaviour of distributed systems, which is simple, intuitionistic, easy to modify, and can also give a complete description of mathematical semantics ${ }^{[1,2,3]}$. The Z.120 $0^{[1]}$ enacted by the International Telecommunication Union has given the standardized definition of Message Sequence Charts. MSC is widely applied in many fields due to its advantages of convenience and intuitive graphic description ${ }^{[4,5]}$. But it also has some disadvantages: The graphical semantic model and mathematical semantic model are both not easy to analyze and simulate ${ }^{[6]}$.

\subsection{Graphical semantic description of MSC}

MSC is composed of objects, including the own event of an object, the message transmission among objects.

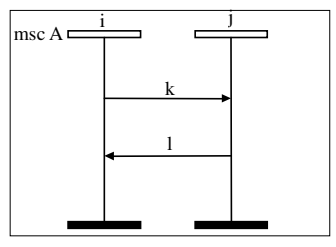

Figure 1 Two messages MSC

As shown in figure 1, the MSC A of two pieces of message is composed of object $i$, object $j$, and message $k$, message $l$. The top and bottom of object $i$ and $j$ are expressed with white rectangle and black rectangle respectively, connecting with straight line which stands for the time. The MSC of two pieces of message shown in figure 1 describe the sequence of the two communication events between object $i$ and object $j$ : Firstly, object $i$ sends a message $k$ to object $j$; secondly, object $j$ receives the message $k$; then object $j$ sends another message $l$ to object $i$; at last, object $i$ receives the message $l$.

\subsection{The process algebraic specification of MSC}

Every MSC can be given a strict description with the process algebraic language, including the algebraic expression of event and the operator. The algebraic expression of event: For example, out $(i, j, k)$ stands for the event that object $i$ sends the message $k$ to object $j$; in $(j, i, k)$ stands for the event that object $j$ receives the message $k$ from object $i$; $\varepsilon$ stands for the state of a object (it doesn't express any action); action(i,a) stands for that object $i$ takes the action a, and so on. The operator: For example, "“" defines strict order, that is the event on the left of this operator takes place precede the event on the right; "+" stands for that to choose a event on either side of this operator to take; “ "| " stands for parallel relation, that is the event on either side of this operator can occur first, and so on.

The priority of the operators above defines as follow: "." is prior to " $\|$ ", and " $\|$ " Is prior to "+ ".

The event sequence of the MSC shown in chart 1 can be expressed with the process algebraic language that: 
$(\operatorname{out}(i, j, k) \| \operatorname{in}(i, j, k)) \cdot(\operatorname{out}(j, i, l) \| \operatorname{in}(j, i, l))$

"+" can be replaced with " $\|$ ". So expression (1) can be replaced with

$(\operatorname{out}(i, j, k) \cdot \operatorname{in}(i, j, k)+\operatorname{in}(i, j, k) \cdot \operatorname{out}(i, j, k)) \cdot(\operatorname{out}(j, i, l) \cdot \operatorname{in}(j, i, l)+\operatorname{in}(j, i, l) \cdot \operatorname{out}(j, i, l))$

Because the message is received only after it is sent out, the expression in $(i, j, k) \cdot \operatorname{out}(i, j, k)$ and in $(j, i, l) \cdot \operatorname{out}(j, i, l)$ should be deleted. So the expression (2) can be simlified to the expression (3) $\operatorname{out}(i, j, k) \cdot \operatorname{in}(i, j, k) \cdot \operatorname{out}(j, i, l) \cdot \operatorname{in}(j, i, l)$

\subsection{The description function of MSC}

The powerful description function of MSC can meet the requirements of general situational modeling. MSC can describe the transmission and receipt of message (shown in char 1). It can also describe the state, action, calculagraph, and meet the function of fuzzy sequence and subsequence charts. Details can be found in paper [2].

In the subsequence function of MSC, every object in the sequence can be abstract object, which is able to extend to a subsequence chart. In this way, we can build a hierarchical model of a system.

\section{The translation to Petri Net}

\subsection{The definition of Petri Net}

Petri $\mathrm{Net}^{[7,8]}$ is a graphical and mathematical modeling tool which can be applied to many systems. It is also a powerful tool to describe and study a complex system characterized by parallel, asynchronism, distributed and random. The definition is as follow:

Petri Net is defined as a three-tuples, $\mathrm{PN}=(\mathrm{P}, \mathrm{T}, \mathrm{F})$, in which $\mathrm{P}$ stands for a set of places which is limited and nonempty, $\mathrm{P}=\left\{p_{1}, p_{2}, \ldots, p_{m}\right\}$;

$\mathrm{T}$ stands for a set of transitions, which is limited and nonempty, $\mathrm{T}=\left\{t_{1}, t_{2}, \ldots, t_{n}\right\}$;

$\mathrm{F}$ stands for a set of connections, $\mathrm{F}=\mathrm{P} \times \mathrm{T} \cup \mathrm{T} \times \mathrm{P} . \mathrm{P}$ and $\mathrm{T}$ also meet the requirement that $\mathrm{P} \cap \mathrm{T}=$ $\Phi$, and $\mathrm{P} \cup \mathrm{T} \neq \Phi$.

In the actual chart, the "place" is expressed with "O", the "transition" is expressed with" $\square$ ", the "connection" is represented by the straight line with arrows, the place and transition are connected with connections.

\subsection{The translation algorithm}

The algorithm of translating the MSC model to Petri Net model is as follows:

Step 1: Divide every object of MSC into three parts, the top, the bottom and the event( sending or receiving the message, state, action and so on);

Step 2: Replace the white rectangle and black rectangle which stand for the top and bottom of a object respectively with " $\circ$ "which stands for the place; 
Step 3: Replace the event( sending or receiving the message, action and so on) with " $\mathbf{-}$ "which stands for the transition;

Step 4: Delete the "state" In the MSC;

Step 5: If in the MSC there are events of fuzzy order, then determine flexibly the order of divided events according to demand;

Step 6: If there are two neighboring transitions on the time line or on the line of message transmission, add a place in the middle of the two transitions;

Step 7: Connect the transition and the place with the connection along the time line and the message transmission line. The direction of the connection must be the same to the direction of the time line and the message transmission line;

Step 8: Translation ends.

\subsection{The illustration of translation algorithm}

According to the translation algorithm, the translation from the MSC of two objects to Petri Net is shown in figure 2 :

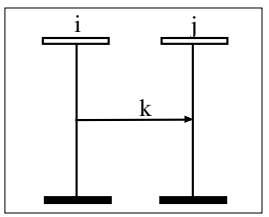

(a) MSC model

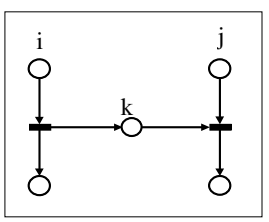

(d) Connecting arc join

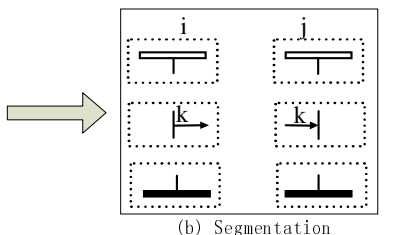

Segmentation

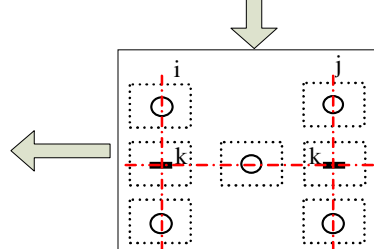

(c) Replace and supplement objects into the MSC corresponding Petri net

\section{Application instance}

\subsection{The description of instance}

Here, we build a model for the procedure from developing new products to coming into the market of an automotive vehicles manufacturer with virtual manufacturing technique.

Details are as follows: When the market is active, the Market_Dept grasps the opportunity to put forward the suggestion of developing new products; Virtual Manufacturing Environment provides the virtual prototype to be evaluated by the Market_Dept; then the Market_Dept feedbacks the evaluation and correction to the Virtual Manufacturing Environment; after the virtual prototype is improved, the Virtual Manufacturing Environment provides the information of the final improved 
products for the Real Manufacturing Environment, which produces the new products in the hundreds. At last, the Market_Dept gets ready for putting the new products on the market.

\subsection{The model of MSC}

\subsubsection{The description of MSC model}

In the model of MSC, the procedure from developing new products to coming into the market is abstracted to be six objects: Market_Environment, Market_Department, Virtual Manufacturing Environment, Real Manufacturing_Environment, Purchase_Department and Error_Manage, which are shown in figure 3 .

\subsubsection{Conflict Resolution Mechanism}

In a more complex system, conflicts are inevitable. Similarly, there will be many conflicts of technique, personnel, resources and so on in the production and business of a manufacturer ${ }^{[9]}$.

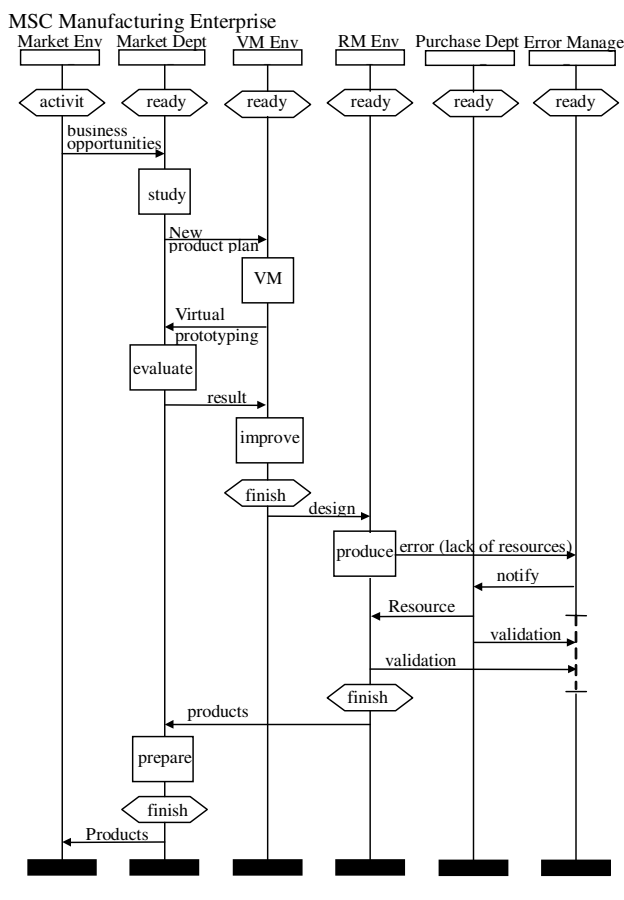

Figure 3 MSC model of manufacturing enterprises

In order to resolve the conflict, in the MSC model of the manufacturer above, we add the Error_Manage. In the actual business operation of a manufacturer, Error_Manage is a flexible department composed of relevant experts and headers of the important department to take realtime communication and coordination, which also integrates the system of experts in charge of conflict resolution with the set of rules, the set of limits and so on to be in charge of resolving and coordinating kinds of conflicts. 


\subsubsection{The instance of Conflict Resolution}

In RM_Env of the model, when it is lack of resources, the Conflict Resolution Mechanism starts: Firstly, it reports to Error_Manage; then Error_Manage reports to Purchase_Dept; after Purchase_Dept supplies resources to RM_Env, it reports confirmation to Error_Manage; meanwhile, after gets the supplement, RM_Env reports the receipt to Error_Manage. Such Conflict Resolution Mechanism makes sure the normal management of a manufacturer.

\subsection{The model of Petri Net}

With the translation algorithm shown in 3.2, the MSC of a manufacturer shown in chart 3 can be translated to the corresponding Petri Net, shown in figure 4.

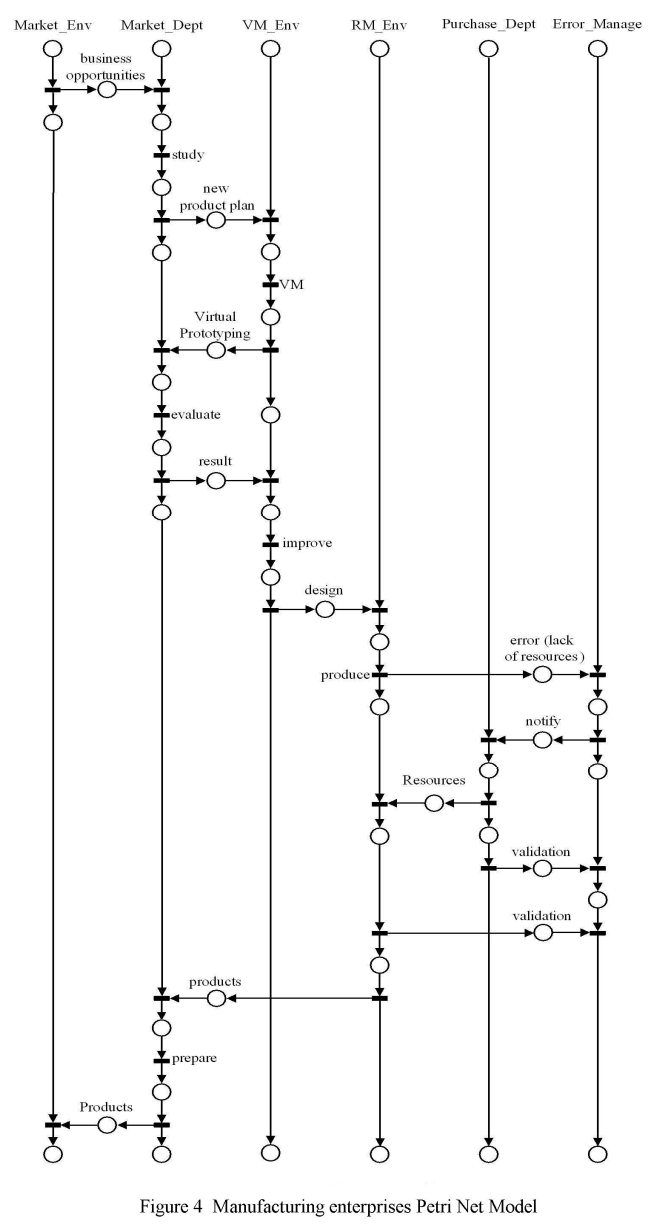

\subsection{The analysis of Petri Net}

Analyze the initialized Petri Net with the existing analytical technique of Petri Net. With respect to safety, it is easy to find that there is no dead lock in the model of Petri Net translated from MSC with the translation algorithm. That is because the message of MSC must be sent out before received. It is won't occur that to receive a piece of message which is can't be sent out, which breaks the necessary condition of the dead lock. So there can't be dead-lock. With respect to 
liveness, the nodes from the beginning to the end are all reachable (the route of message transmission is attainable). Therefore, the Petri Net is safe and feasible.

\section{CONCLUSION}

In the Petri Net modeling of a complex system, it has many advantages to get the Petri Net model from MSC model:

(1) Because of the powerful function of the description of MSC, it is easy to build a simple, intuitionistic MSC model of a complex system;

(2) The subsequence charts function of MSC support the theory of hierarchical modeling and module abstract and composite ${ }^{[10]}$;

(3) The translation algorithm provides a powerful tool to translate the MSC model to Petri Net model;

(4) MSC model can help to understand Petri Net model of a complex system, enhance the readability of Petri Net model, and avoid the dead lock effectively;

(5) It can modify Petri Net model by modifying the responding MSC model, which makes building and modifying Petri Net model based on the simple and intuitionistic MSC model.

After Petri Net model is built, how to evaluate a complex system with the responding Petri Net model is the next target of author's group.

\section{ACKNOWLEDGEMENTS}

This work is supported by the Natural Science Foundation of China (NSFC) under Grant No. 60434010 and No. 61174144. And thanks professor Fang-zhou BAI for his valuable suggestions.

\section{REFERENCES}

[1] Geneva:ITU-T, (1996). ITU-T Recommendation Z.120: Message Sequence Chart (MSC) .

[2] S.Mauw, (1996). The formalization of Message Sequence Charts. Computer Networks and ISDN Systems. No.28,pp1643-1657.

[3] Rudolph, E., J. Grabowski and P. Graubmann,( 1996). Tutorial on Message Sequence Charts. Computer Networks and ISDN Systems. No.28,pp629-1641.

[4] Kumar, Sandeep; Khoo, Siau-Cheng; Roychoudhury, Abhik; Lo, David,(2011). Mining message sequence graphs. 33rd International Conference on Software Engineering, ICSE 2011, May 21, 2011 - May 28, 2011, p 91-100.

[5] Dan, Haitao; Hierons, Robert M.(2011), Conformance testing from message sequence charts. Proceedings - 4th IEEE International Conference on Software Testing, Verification, and Validation, ICST 2011, p 279-288.

[6] Olaf Kluge, ( 2003). Modelling a Railway Crossing with Message Sequence Charts and Petri Nets. Lecture Notes in Computer Science. Springer Verlag. No.2472,pp197-218. 
[7] T.Murata,( 1989). Petri Nets: Properties, Analysis and pplications. Proceedings of the IEEE. No.77,pp541-580.

[8] Chongyi Yuan,( 1998). Principle of Petri Net. Beijing:Electronics Industrial Press.

[9] Song Huang, Yujin Hu,Chenggang Li,( 2004). A TCPN based approach to model the coordination in virtual manufacturing organizations. Computers \& Industrial Engineering. No.47,pp61-76.

[10] Zhibin Jiang,( 2004). Petri Net and its application in the manufacturing system modeling and control. Beijing: Machinery Industrial Press.

\section{Authors}

Chen-xi SHAO, male, 1954, M.Eng., associate professor, research interests are simulation of complex system and qualitative simulation;

Zhen-zhong SHAO, male,1983, B. Eng., engineer, research intersts are CIM, $\mathrm{CAD} / \mathrm{CAM}$ and VR.

Wei-hua WANG, female, 1960, B. In.\& Co., research interests are MRP-II, ERP andSCM.
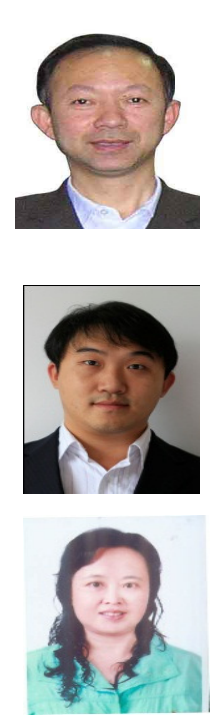